\title{
COMPARATIVE ANALYSIS OF RISAT-1 AND SIMULATED RADARSAT-2 HYBRID POLARIMETRIC SAR DATA FOR DIFFERENT LAND FEATURES
}

\author{
Vineet Kumar $^{\text {a, }}$, Y. S. Rao ${ }^{\text {a }}$ \\ ${ }^{\text {a }}$ Centre of Studies in Resource Engineering, Indian Institute of Technology Bombay, 400076 Mumbai, India \\ vineetk008@gmail.com
}

Commission VIII/Mid Term Symposium-2014

KEY WORDS: Hybrid Polarimetry, RISAT-1, RADARSAT-2, Stokes Parameters, CPR

\begin{abstract}
:
The purpose of this study is to compare the performance of first hybrid polarimetric spaceborne satellite RISAT-1 data and simulated hybrid polarimetric data from quad-pol RADARSAT-2 data for different land use land cover (LULC) classes. The present study compares Stokes (g0, g1, g2 and g3) and its decomposed parameters (m, chi, delta and CPR) for satellite data acquired from RISAT1 and RADARSAT-2 over Vijayawada, Andhra Pradesh, India. Further, backscattering coefficients are also compared for different LULC types. The results indicate that both the satellites are following approximately the same trend for different classes except for settlements in RISAT-1.
\end{abstract}

\section{INTRODUCTION}

In recent years, compact polarimetry has demonstrated its potential in the field of radar remote sensing for earth observation. Compact polarimetry SAR imaging systems have advantages over conventional single and dual polarized SAR systems. Compact pol SAR systems also have advantages over quad-pol SAR systems in terms of hardware performance (wide swath coverage and half transmitted power requirement capabilities) (Souyris et al., 2005 and Raney 2007). In compact polarimetry, only one polarization is transmitted and two orthogonal polarizations are received. Hybrid polarimetry is a special case of compact polarimetry with circular (right/left) polarization transmission and dual linear $(\mathrm{H} \& \mathrm{~V})$ polarization reception. India's RISAT-1 is the first spaceborne hybrid polarimetry operating satellite dedicated for earth observation, operating in right circular transmission and dual linear reception mode. In the near future hybrid polarimetric SAR data will also be available from ALOS-2 and RADARSAT constellation mission (RCM) satellites. To evaluate the potential of compact polarimetry mode, earlier investigations have been conducted by using simulated spaceborne or airborne quad-pol SAR data (Ainsworth et al., 2009 and Charbonneau et al., 2010). Stokes and its decomposed parameters were used for land cover classification, mapping and crop assessment (Brisco et al., 2012 and Ballester-Berman and Lopez 2012). Some initial studies have been conducted with RISAT-1 data for crop acreage estimation, classification and oceanographic applications (Chakraborty et al., 2013, De et al., 2014). In this study we try to evaluate the comparative performance of real spaceborne hybrid polarimetric data with simulated hybrid polarimetric data for various land covers using Stokes and its derived parameters.

\section{STUDY AREA AND DATASETS}

The study area is a part of Vijayawada city and its surroundings in the Andhra Pradesh state of India. It is located at $16^{\circ} 31^{\prime} \mathrm{N}$ and $80^{\circ} 39^{\prime} \mathrm{E}$ along the Krishna river coast. The major land use land cover of the study area comprises of agricultural fields, water body, settlement and sand in the river bed. Settlement-1 and Settlement- 2 are oriented and non-oriented man-made structures respectively; orientation is with respect to the direction of line of sight of RADAR (Lee \& Ainsworth, 2011). Rice, cotton, banana, sugarcane and corn are main crops cultivated in the month of March. For this study rice, cotton and banana are taken under consideration. In the study area rice crop is grown twice in a year. During the field visit it has been observed that rice crop was at late tillering stage while cotton was matured with dried leaves. The majority of rice and cotton fields size are more than $100 \mathrm{~m} \times 100 \mathrm{~m}$. Single look complex (SLC) satellite data product of RADARSAT-2 and RISAT-1 were acquired over the study area and field work was carried out synchronously. The acquired scenes are of two days gap with approximately $4^{0}$ difference in the scene centre incidence angle. Detailed technical configurations of datasets are given in Table1.

\begin{tabular}{|l|c|c|}
\hline $\begin{array}{c}\text { Satellite/ } \\
\text { Parameters }\end{array}$ & RADARSAT-2 & RISAT-1 \\
\hline Date of Acquisition & 11-March-2014 & 09-March-2014 \\
\hline Frequency & $\begin{array}{c}\text { C-Band } \\
(5.405 \mathrm{GHz})\end{array}$ & $\begin{array}{c}\text { C-Band } \\
(5.35 \mathrm{GHz})\end{array}$ \\
\hline Pass & Ascending & Ascending \\
\hline Look & Right & Left* \\
\hline Beam Mode & FQ 8 & FRS 1 \\
\hline Polarization & $\mathrm{HH}, \mathrm{HV}, \mathrm{VH}, \mathrm{VV}$ & RH, RV \\
\hline Incidence Angle & $27.20^{\circ}$ & $31.34^{\circ}$ \\
\hline $\begin{array}{l}\text { Input Resolution } \\
\text { (along x across) }\end{array}$ & $\begin{array}{c}5.2 \times 7.6 \\
(\mathrm{~m} \times \mathrm{m})\end{array}$ & $\begin{array}{c}3.33 \times 2.34 \\
(\mathrm{~m} \times \mathrm{m})\end{array}$ \\
\hline $\begin{array}{l}\text { Output Spacing } \\
\text { (Line x Pixel) }\end{array}$ & $\begin{array}{c}4.73 \times 4.79 \\
(\mathrm{~m} \times \mathrm{m})\end{array}$ & $\begin{array}{c}1.8 \times 2.36 \\
(\mathrm{~m} \times \mathrm{m})\end{array}$ \\
\hline
\end{tabular}

Table 1. Technical parameters of satellite data acquired (* indicate right looking in international terminology)

\section{METHODOLOGY}

RADARSAT- 2 and RISAT- 1 scenes are multilooked $2 \times 1$ and $4 \times 3$ in azimuth and range direction respectively followed by a refined Lee filter with a kernel size of $5 \times 5$. The multilook is done to bring both data sets into same resolution. RADARSAT2 scene was used as for geocoding using ASF Map ready tool. 
Manual image-to-image registration of RISAT-1 scene with RADARSAT-2 scene has been performed in ENVI with RMSE error less than 0.5 pixel. Simulated hybrid pol data was generated from RADARSAT-2 quad pol product with right and left circular polarization assumptions. The simulated hybrid polarimetric data are represented in terms of Stokes vector format. From Stokes vector, specific compact polarimetric parameters; degree of polarization (DoP), ellipticity angle (chi) and relative phase (delta) along with circular polarization ratio (CPR) were also extracted from both the datasets. Further, mean and standard deviation values of Stokes parameters (g0, g1, g2 and g3) and (DoP, chi, delta and CPR) were plotted for quantitative comparison. In addition, backscattering coefficient $\left(\sigma^{\circ} \mathrm{RH}, \mathrm{RV}\right)$ in circular polarization mode were extracted for quantitative analysis. Here left and right circular transmission polarization sign convention for simulated RADARSAT-2 data is on the basis of PolSARpro software.

\section{STOKES PARAMETERS AND ITS DERIVED PRODUCTS}

In 1852 Gabriel Stokes introduced four parameters collectively known as Stokes vector to completely describe a polarized wave. A stokes vector can describe quantitatively a fully polarized wave as well as a partial polarized wave. For a given incident polarization (left/right circular or pi/4) with a linear polarization basis Stokes parameters are givens as (Raney 2007 and Shirvaney 2012)

$\left[\begin{array}{l}g_{0} \\ g_{1} \\ g_{2} \\ g_{3}\end{array}\right]=\left[\begin{array}{c}<\left|E_{H}\right|^{2}+\left|E_{V}\right|^{2}> \\ <\left|E_{H}\right|^{2}-\left|E_{V}\right|^{2}> \\ 2 \operatorname{Re}<E_{H} E_{V}{ }^{*}> \\ 2 I m<E_{H} E_{V}{ }^{*}>\end{array}\right]$

Where $\langle\ldots>$ indicates spatial averaging, $E$ is complex electric field of received polarization and "*" refers to complex conjugate of the number. The first Stokes parameter $g_{0}$ measures the total amount of energy in the wave while other three describes its state of polarization. From the Stokes parameters it is possible to further extract its decomposed (child) parameters such as degree of polarization (DoP), ellipticity angle (Chi), relative phase (Delta) and circular polarization ratio (CPR) which are useful in quantitative measurement of received wave. DoP expresses the polarization state of received electromagnetic wave with value ranging from 0 to 1 and is given as

DoP $=m=\frac{\sqrt{g_{1}^{2}+g_{2}^{2}+g_{3}^{2}}}{g_{0}^{2}}$

For completely polarized waves DoP value is 1 while for completely unpolarized wave is at 0 . The chi angle (eq.3) defines amount of polarization of partially polarized wave.

Chi $=\mu_{E}=\frac{g_{3}}{g_{0}}$

Delta is the relative phase between two received electric field vectors and varies between $-180^{\circ}$ to $+180^{\circ}$.

Delta $=\delta=\operatorname{atan}\left(\frac{g_{3}}{g_{2}}\right)$

CPR is the ratio of difference of Stokes first and fourth parameter to the sum of these two and gives the information about sense of circular polarization (Campbell 2012).

$\mathrm{CPR}=\mu_{c}=\frac{\left(g_{0}-g_{3}\right)}{\left(g_{0}+g_{3}\right)}$

\section{RESULTS AND DISCUSSION}

For visual interpretation of various land features, the colour composite RGB image of RISAT-1 and simulated hybrid polarimetric image of RADARSAT-2 of the study area are shown in figure 1 . The red/pink colour appearance of targets shows vertically oriented features like rice crop, settlement etc. The surface features (bare fields, water body and sand) appear at different scale of blue colour in the RISAT-1 and RADARSAT-2 left circular (LC) RGB images while in reddish tone in RADARSAT-2 right circular (RC) image. The green colour indicates the trees and non-oriented settlements with the dominance of volume scattering component. It may be observed from figure 1 that RISAT- 1 right circular m-chi decomposed RGB colour matches with RADARSAT-2 left circular RGB due to sign convention taken in PolSARpro software.

A relative trend of mean values of Stokes parameter for RISAT1 and RADARSAT-2 left and right circular polarization is shown in figure 2 . The trend observed for all classes is same except the settlement-1. As first Stokes parameter figure 2(a) gives information about total intensity of wave so there is no difference observed in simulated RADARSAT-2 (RC and LC) and RISAT-1 extracted values. But because of difference in incidence angles and other system parameters a difference in mean of intensity values are reported. In case of water bodies where specular reflection is dominant and incident angle does not play a significant role in backscatter wave, the value of Stokes first parameter is nearly same in both cases.

The first three Stokes parameter (g0, g1, g2) values are very high for settlement-1 type urban areas, while for fourth Stokes parameter (g3) value was very low compared to observations of simulated RADARSAT-2 data. The reason may be due to the calibration of hybrid polarimetric hardware architecture or saturation of satellite sensor for high backscatter dihedral targets for RISAT-1 Mean and standard deviation values of various quantitative compact decomposition measures extracted from Stokes parameters are represented in figure 3 for both the datasets. Vertical bars at mean data are standard deviation.

Degree of polarization (DoP), which is a measure of the amount of polarization from targets, follows approximately similar trend for different land covers. DoP values were found closer for banana, cotton, settlement-1, 2 and tree classes, whereas for surface features (water, bare \& sand) and rice crop, significant difference has been observed as shown in figure 3(a). For features with high volume scattering, DoP values were observed to be low (near 0.1). But exceptionally in case of RISAT-1 data, water body also indicates lower DoP due to roughness.

Ellipticity angle (Chi) provides information about polarized part of quasi-monochromatic wave. The ellipticity angle has similar mean values from RISAT- 1 and simulated RADARSAT- 2 left circular polarized (LC) data for all classes except banana and trees as shown in figure 3(b). Approximately, $30^{\circ}$ angular difference is observed for banana and tree class in both the datasets. Figure 3(c) shows mean and standard deviations (vertical bars) of relative phase (delta) values. Standard deviations are higher for all features. Hence, there is no clear discrimination among different classes. Figure 3(d) shows circular polarization ratio (CPR) for various surface features. For bare field, water body and sand, CPR value is less than 0.5 for RISAT-1 and RADARSAT-2 LC polarized data, while for other features CPR values are higher. Opposite trend is observed in RADARSAT-2 RC polarized data. 


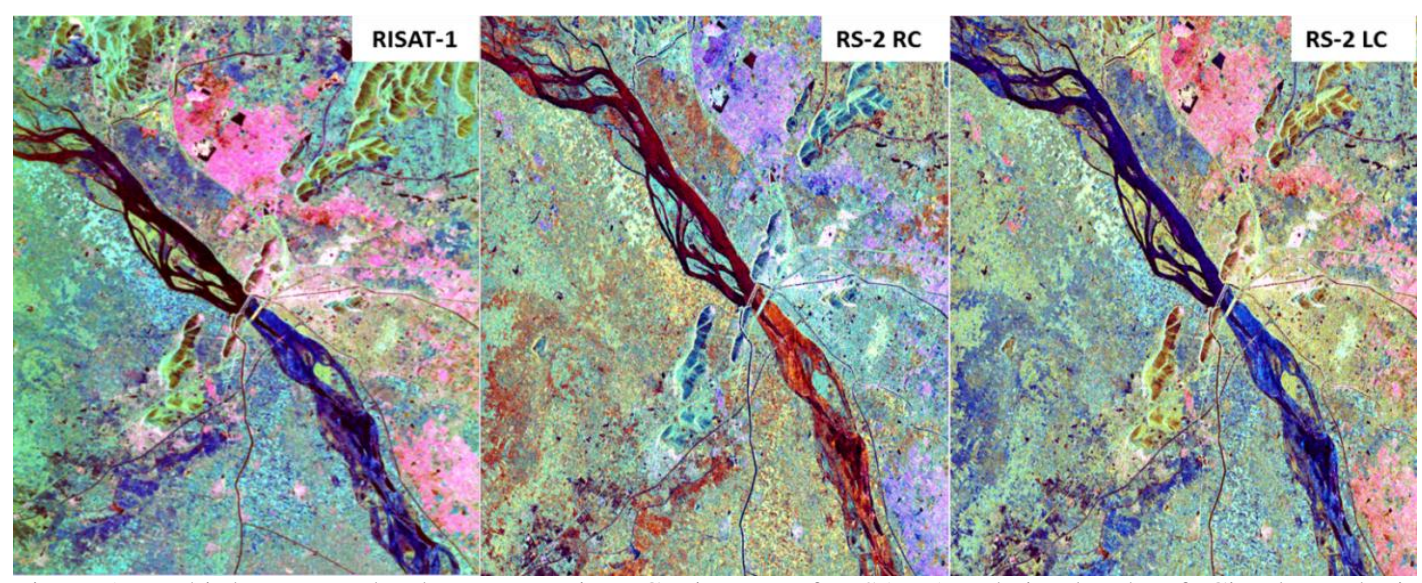

Figure 1. m-chi decomposed colour composite RGB images of RISAT-1 and simulated Left Circular and Right Circular Polarized RADARSAT-2 data.
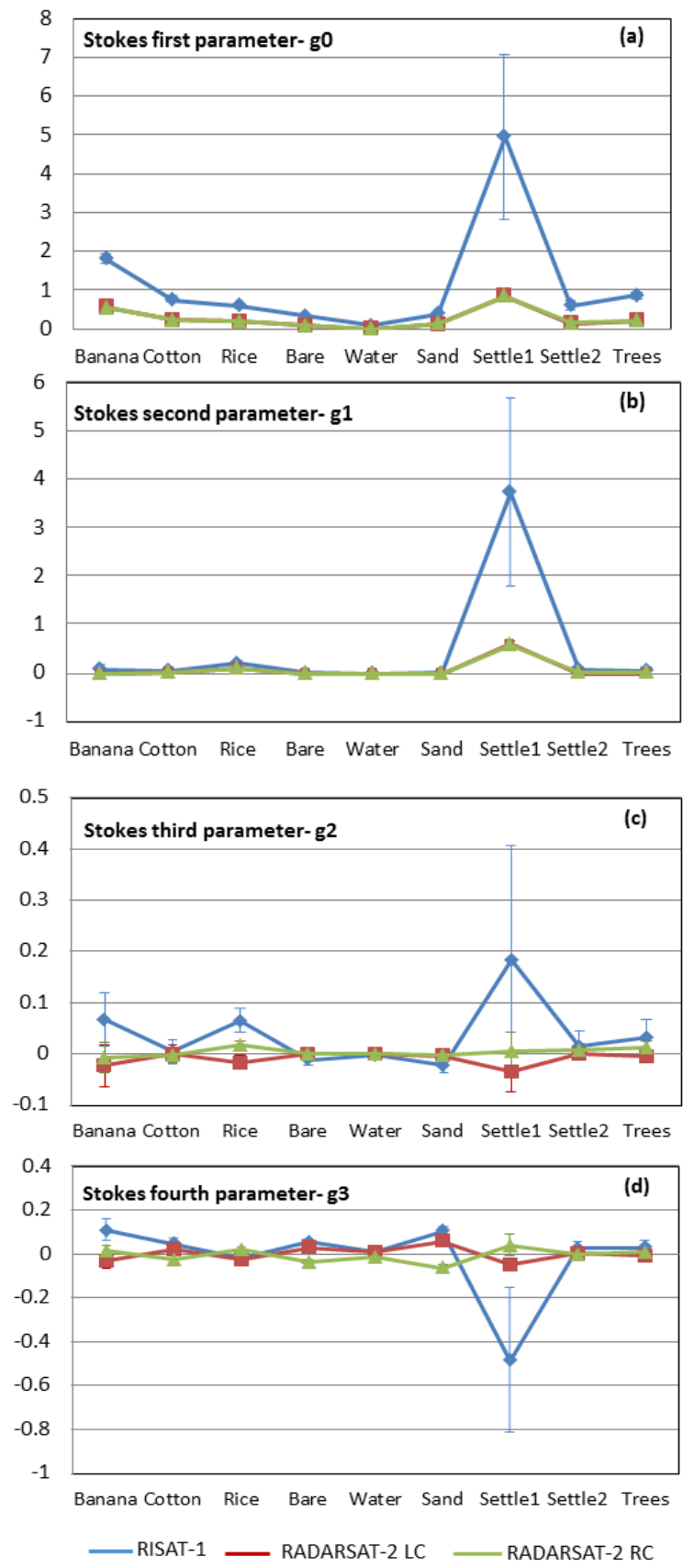

Figure 2. Stokes parameters for different LULC types.
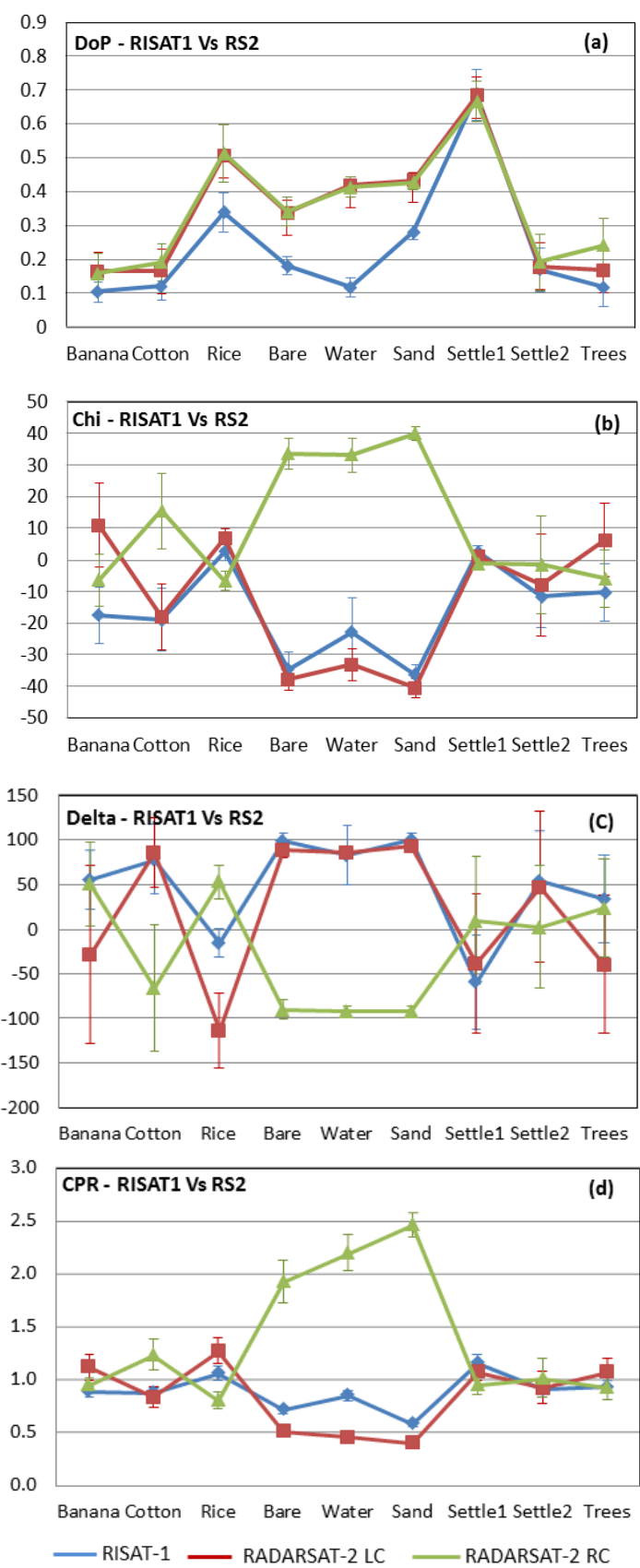

Figure 3. Stokes derived parameters for different LULC types. 
Table 2 indicates the comparison of average backscattering coefficient $\left(\sigma^{\circ}\right)$ values in right circular transmission and linear received polarization $(\mathrm{H}, \mathrm{V})$ for different features. Settlement-1 type urban areas which are oriented towards radar look direction gives very high backscatter coefficient (around $6 \mathrm{~dB}$ ) in RH polarization mode of RISAT-1 data while in RADARSAT-2 the values is around $-1.30 \mathrm{~dB}$, A relative difference of 5 to $6 \mathrm{~dB}$ was observed for both the satellite data configurations in all identified LULC features, which may be because of difference in noise floor levels or calibration constants of RISAT-1 and RADARSAT-2.

\begin{tabular}{|l|c|c|c|c|}
\hline LULC features & RISAT-1 & $\begin{array}{c}\text { Simulated } \\
\text { Radarsat-2 }\end{array}$ & RISAT-1 & $\begin{array}{c}\text { Simulated } \\
\text { Radarsat-2 }\end{array}$ \\
\hline Polarization & $\mathrm{RH}(\mathrm{dB})$ & $\mathrm{RH}(\mathrm{dB})$ & $\mathrm{RV}(\mathrm{dB})$ & $\mathrm{RV}(\mathrm{dB})$ \\
\hline Banana & -0.23 & -5.54 & -0.56 & -5.18 \\
\hline Cotton & -3.90 & -8.86 & -4.38 & -9.41 \\
\hline Rice & -3.83 & -7.91 & -6.73 & -12.55 \\
\hline Bare Fields & -7.45 & -12.93 & -7.64 & -12.83 \\
\hline Water Body & -12.63 & -19.36 & -12.46 & -18.11 \\
\hline Sand & -6.79 & -11.46 & -6.91 & -11.23 \\
\hline Settlement-1 & 6.38 & -1.30 & -2.17 & -8.71 \\
\hline Settlement-2 & -4.63 & -10.95 & -5.74 & -10.77 \\
\hline Trees & -3.25 & -9.68 & -3.79 & -9.49 \\
\hline
\end{tabular}

Table 2. Estimated Backscatter contributions in Right circular transmit and horizontal (RH) and vertical (RV) receive respectively.

\section{CONCLUSIONS}

The potential of spaceborne hybrid polarimetry satellite RISAT1 and simulated RADARSAT-2 data is explored for LULC discrimination. The relative variation of Stokes and its decomposed parameters values for different feature class in both the satellite products follows same trend except settlement-1 class. This initial study demonstrates the potential use of RISAT-1 hybrid polarimetric SAR data for LULC analysis and other applications with fewer exceptions.

\section{ACKNOWLEDGEMENT}

The authors thank MDA-GSI and Canadian Govt. for providing RADARSAT-2 data through project code SOAR-EI-5174.

\section{REFERENCES}

Ainsworth, T. L., Kelly, J. P., \& Lee, J. S., 2009. Classification comparisons between dual-pol, compact polarimetric and quadpol SAR imagery. ISPRS Journal of Photogrammetry and Remote Sensing, 64(5), pp. 464-471.

Ballester-Berman, J.D.; Lopez-Sanchez, J.M., 2012. Time Series of Hybrid-Polarity Parameters over Agricultural Crops, IEEE Transactions on Geoscience and Remote SensingLetters, vol.9, no.1, pp.139-143.

Brisco, B., Li, K., Tedford, B., Charbonneau, F., Yun, S., \& Murnaghan, K. (2013). Compact polarimetry assessment for rice and wetland mapping. International Journal of Remote Sensing, 34(6), 1949-1964.
Campbell, B. A., 2012. High circular polarization ratios in radar scattering from geologic targets, Journal of Geophysical Research: Planets (1991-2012), 117 (E6)

Chakraborty, M., Panigrahy, S., Rajawat, A. S., Kumar, R., Murthy, T. V. R., Haldar, D. \& Kundu, S., 2013. Initial results using RISAT-1 C-band SAR data, Current Science, 104(4), pp.490-501

Charbonneau, F. J., B. Brisco, R. K. Raney, H. McNairn, C. Liu, P. W. Vachon, J. Shang et al., 2010. Compact polarimetry overview and applications assessment, Canadian Journal of Remote Sensing 36, no. sup2: S298-S315.

De, S., Kumar, V., \& Rao, Y. S., 2014. Crop Classification using RISAT-1 Hybrid Polarimetric SAR Data, In EUSAR 2014; 10th European Conference on Synthetic Aperture Radar; Proceedings of (pp. 1-4). VDE.

Raney, R. K., 2007. Hybrid - polarity SAR architecture. IEEE Transactions on Geoscience and Remote Sensing, 45(11), pp. 3397-3404.

Raney, R. K., Cahill, J. T., Patterson, G., \& Bussey, D. B. J. 2012. The m-chi decomposition of hybrid dual polarimetric radar data with application to lunar craters, Journal of Geophysical Research: Planets (1991-2012), 117(E12).

Lee, J. S., \& Ainsworth, T. L. 2011. The effect of orientation angle compensation on coherency matrix and polarimetric target decompositions. IEEE Transactions on Geoscience and Remote Sensing, 49(1), 53-64.

Shirvaney, R., 2012. Estimation of Degree of Polarization in Polarimetric SAR imagery: Principles and Applications $P h D$. Dissertation, University of Toulouse, 2012

Souyris, J. C., Imbo, P., Fjortoft, R., Mingot, S., \& Lee, J. S. 2005. Compact polarimetry based on symmetry properties of geophysical media: The $\pi / 4$ mode. IEEE Transactions on Geoscience and Remote Sensing, 43(3), 634-646. 